\title{
Tratamiento de Lixiviados de Rellenos SANITARIOS: Avances Recientes
}

\section{Eugenio Giraldo*}

\section{INTRODUCCIÓN}

Enterrar los residuos sólidos urbanos ha sido, y es aún, la práctica más utilizada por las sociedades del mundo para su manejo. A pesar de la creciente conciencia mundial sobre la necesidad de Reducir, Reusar y Reciclar los materiales que fluyen a través de la sociedad, la implementación real de estas políticas ha encontrado numerosos obstáculos que han impedido su materialización en hechos concretos. Parte del problema se encuentra en la poca internalización de los costos ambientales en que se incurre en la producción de bienes que finalmente se descartan convirtiéndose en residuos. La comparación final sobre que hacer con un bien descartado se hace en términos de las alternativas para su manejo final, -mas no en los impactos ambientales que generó su producción, distribución y uso-, siendo con frecuencia la alternativa más económica su disposición en un relleno sanitario. En sociedades en donde el costo del capital es alto, como en Latinoamérica, y en donde existen numerosas necesidades insatisfechas que compiten por los recursos, con frecuencia terminan los rellenos sanitarios siendo las opciones mas utilizadas a pesar de la clara conciencia de que las prioridades las fijan las tres R. Colombia, por ejemplo, en su política nacional de residuos sólidos ha adoptado como prioridad nacional las tres R. Reducción, Reuso y Reciclaje en ese orden.

Una vez se han enterrado los residuos sólidos es necesario minimizar los impactos de esta práctica. Para empezar, el agua que ha entrado en contacto con la basura recoge gran cantidad de las sustancias que originalmente estaban dentro del residuo, quedando de esa manera altamente contaminada. Esta agua se denomina lixiviado, y es uno de los líquidos más contaminados y contaminantes que se conozcan. De no recogerse adecuadamente y luego tratarse, el lixiviado puede conta- minar a su vez aguas subterráneas, aguas superficiales y suelos. Por esta razón, y para evitar que esto ocurra, los rellenos sanitarios se impermeabilizan, se drenan apropiadamente y los lixiviados recogidos por estos drenes, se deben tratar.

La presente contribución revisa las tecnologías recientes para el tratamiento de lixiviados de rellenos sanitarios. Primero se hace una presentación de las principales características de los lixiviados desde el punto de vista del tratamiento que se pretende hacer, luego se hace una presentación crítica de las tecnologías que se han desarrollado recientemente para el tratamiento de los lixiviados de rellenos sanitarios. Se concluye con unas recomendaciones sobre las consideraciones que deben tenerse en cuenta a la hora de la selección de la tecnología del tratamiento de los lixiviados de los rellenos sanitarios. No se pretende de ninguna manera mostrar de manera exhaustiva y detallada la evolución de las tecnologías para el tratamiento de los lixiviados, sino hacer una discusión de aquellas más recientes.

\section{Características de los Lixiviados que Afectan su \\ TRATAMIENTO}

Existen numerosas caracterizaciones de los lixiviados en donde se hace énfasis en su alto poder contaminante ${ }^{1}$. Se concluye usualmente que los lixiviados contienen toda característica contaminante principal, es decir, alto contenido de materia orgánica, alto contenido de nitrógeno y fósforo, presencia abundante de patógenos e igualmente de sustancias tóxicas como metales pesados y constituyentes orgánicos. Estas características son importantes en cuanto nos indican qué es lo

* Ingeniero Civil, Universidad de Los Andes. MSc. Ingeniería Ambiental y Ph. D. en Ingeniería Ambiental, University of Massachussets, USA. Profesor Asociado, Departamento de Ingeniería Civil y Ambiental, Universidad de Los Andes. 
que toca removerle a los lixiviados durante su tratamiento, sin embargo, desde el punto de vista de la selección de la tecnología existen otras características que, sin ser necesariamente contaminantes, pueden afectar el funcionamiento de los procesos de tratamiento. A continuación se revisan las características de los lixiviados desde la perspectiva del sistema de tratamiento que se piensa seleccionar.

\subsection{Calidad de los lixiviados}

La calidad de los lixiviados en un relleno sanitario varía grandemente en el tiempo, al igual que con el tipo de relleno sanitario que se tenga. En particular vale la pena mencionar las diferencias que se tienen en las calidades de los lixiviados entre aquellos de los países desarrollados con los de los países en vía de desarrollo. De manera resumida se puede decir que los lixiviados de los rellenos sanitarios de los países en desarrollo presentan concentraciones mucho mayores de $\mathrm{DBO}$, amoníaco, metales y sustancias precipitables que aquellos de países desarrollados. Como se verá a continuación esto tiene importantes implicaciones para la operatividad y el rendimiento de los procesos de tratamiento, y debe tenerse cautela cuando se busque hacer la adaptación de las tecnologías a los casos locales.

Las diferencias se originan principalmente en los altos contenidos de materia orgánica fácilmente biodegradable, MOFBD, que se tiene en los residuos sólidos en los países en desarrollo. La MOFBD tiene un contenido de humedad alto, y como su nombre lo indica se degrada rápidamente en el relleno sanitario, produciendo a su vez altas concentraciones de ácidos grasos volátiles y de amoníaco- en general mucho más altas que las que se reportan típicamente para lixiviados de países desarrollados- producto de la fermentación inicial. A su vez, estos ácidos se diluyen fácilmente en el lixiviado del relleno sanitario, le bajan el $\mathrm{pH}$ y contribuyen a la solubilización de los metales presentes en los residuos dispuestos en el relleno.

Como consecuencia los lixiviados de las áreas de los rellenos sanitarios que han sido recientemente rellenadas producen un lixiviado altamente contaminante, denominado lixiviado joven. A partir de ese momento, las concentraciones de las sustancias en el lixiviado de una cochada de basura en el relleno sanitario disminuyen continuamente en el tiempo, esto ocurre como regla general, más sin embargo, en algunos casos como metales que presentan reacciones de óxido-reducción, puede ocurrir que la concentración al inicio del proceso de lixiviación no sea la mayor. Sin embargo, teniendo en cuenta que un relleno sanitario se opera por lustros o décadas, siempre va a haber una parte del relleno que aporta lixiviado joven, la que se esta rellenando en ese momento, mientras que otras partes del relleno tienen lixiviado maduro, las que tienen unos años, y otras lixiviado viejo, las que tienen mas de cinco años. En la Tabla 1 se resume las principales características de los lixiviados jóvenes y viejos en un relleno sanitario.

TABLA 1.

Comparación de Características Típicas de los Lixiviados DE RELLENOS SANITARIOS

\begin{tabular}{|l|l|l|}
\hline CARACTERÍstica & Lixiviado Joven & LiXiviado VIEJO \\
\hline DBO & Muy alto & Bajo \\
\hline DQO & Muy alto & Alto \\
\hline Amoniaco & Muy alto & Alto \\
\hline Fósforo & $\begin{array}{l}\text { Usualmente } \\
\text { Deficiente (1) }\end{array}$ & Suficiente \\
\hline pH & Muy bajo & Bajo \\
\hline Detergentes & Muy Altos & Bajos \\
\hline Sales disueltas & Muy Altas & $\begin{array}{l}\text { Bajas (relati } \\
\text { vamente) }\end{array}$ \\
\hline $\begin{array}{l}\text { Agentes Incrus- } \\
\text { tantes (Fe, Ca, Mg) }\end{array}$ & Muy Altos & Bajos \\
\hline Metales Pesados & Muy Altos & Bajos \\
\hline
\end{tabular}

(1) Deficiente desde el punto de vista de un tratamiento biológico aerobio

Como se puede observar por las características de la Tabla 1 el tipo de problema del tratamiento que se enfrenta con un lixiviado joven y uno viejo es muy diferente. Para empezar, las concentraciones de todos los parámetros son mucho mayores en un lixiviado joven que en un lixiviado viejo. Por ejemplo, la relación DBO / DQO para un lixiviado joven es alta, indicando una buena biodegradabilidad, mientras que para un lixiviado viejo es baja indicando una pobre biodegradabilidad de la materia orgánica. Las concentraciones de sales disueltas, y metales pesados son mucho mayores en un lixiviado joven, generando problemas de toxicidad en el caso de que se quieran utilizar procesos biológicos para la remoción de la DBO. Por otra parte, las concentraciones de agentes incrustantes como el hierro, el calcio y el magnesio, generan grandes problemas prácticos pues taponan la mayoría de los conductos, tuberías, accesorios, válvulas, membranas, tanques etc. en donde el lixiviado entra en contacto con el sistema de conducción y de tratamiento. El atascamiento de 
los sistemas de drenaje, de conducción y de tratamiento de los lixiviados es siempre un problema práctico de gran significancia en los rellenos sanitarios. El bajo $\mathrm{pH}$ a su vez puede interferir con el funcionamiento de numerosas tecnologías que se proponen, por ejemplo, la volatilización del amoníaco, para la cual se requieren $\mathrm{pH}$ básicos, o con algunos procesos biológicos como la nitrificación, o químicos como la oxidación del hierro.

De igual manera, la remoción de DBO se ve afectada por la toxicidad que generan los metales, pero a su vez, la remoción de metales, incluyendo aquellos incrustantes como el hierro, se ve interferida por la presencia de la DBO que sirve como agente acomplejante que mantienen los metales en solución dificultando y limitando severamente su remoción. Estamos entonces en una encrucijada, los metales afectan la remoción de la DBO, y la presencia de DBO la remoción de los metales, sin dejar en claro por donde empezar a tratar.

Con respecto a la presencia de altos contenidos de nitrógeno, los lixiviados jóvenes pueden llegar a generar problemas de toxicidad por amoníaco en los sistemas biológicos anaerobios para la remoción de DBO. Por otra parte, el fósforo, que como valor absoluto se encuentra en altas concentraciones, con frecuencia se encuentra insuficiente en los lixiviados jóvenes a la hora de la remoción de la DBO por procesos biológicos, lo que lleva a la necesidad de tener que adicionar fósforo en el proceso. En el caso de los lixiviados viejos el fósforo usualmente no es un factor limitante.

La alta presencia de detergentes, por otra parte, presenta problemas prácticos importantes a la hora de utilizar procesos biológicos. Si se utilizan procesos aerobios, en donde es necesario introducir aire al agua por medio de sistemas de inyección, la formación de espumas en los tanques de aireación genera problemas operativos difíciles de manejar. En el caso de sistemas anaerobios, en donde se produce biogás en gran cantidad, igualmente se pueden formar espumas que interfieren con el funcionamiento normal del proceso.

Como se puede observar los lixiviados presentan numerosos problemas que deben tenerse en cuenta al momento de seleccionar la tecnología de tratamiento.

\subsection{Cantidad de los Lixiviados}

La cantidad de los lixiviados en un relleno sanitario también es un punto importante a considerar en el momento de la selección de la tecnología para su tratamiento. La cantidad de los lixiviados es función de tres variables principales. El área rellenada, la cantidad de infiltración que se permita, y el sistema de drenaje, imper- meabilización. El área rellenada afecta porque es a través de ella que se realiza la entrada y el contacto del agua de infiltración con la basura. $\mathrm{Al}$ aumentar el área rellenada, aumenta paralelamente la cantidad de lixiviados. Teniendo en cuenta que los rellenos son sistemas que duran lustros y décadas en su funcionamiento, igualmente se espera que la cantidad de lixiviados aumente. Este aumento es un aumento gradual, lento, con los años.

Por otra parte la cantidad de infiltración que se permita al relleno depende de numerosas variables tales como: la operación que se le dé al relleno- como por ejemplo la desviación de aguas de escorrentía-, la cantidad de precipitación directa que se tenga en la zona, y la presencia de infiltraciones subterráneas. Usualmente las variaciones en la producción de lixiviados asociadas a las infiltraciones, son variaciones rápidas, asociadas a la lluvia por ejemplo, y que hacen oscilar notablemente la cantidad de lixiviados que se debe tratar. Por ultimo, los sistemas de drenaje e impermeabilización son importantes porque son los que permiten que los lixiviados no contaminen los suelos y las aguas subterráneas, y además que el lixiviado que se produce en realidad se recoja y se permita su tratamiento. De la misma manera, una vez se hace el cerramiento de las diferentes áreas de los rellenos, los caudales de infiltración disminuyen notablemente, generando una caída abrupta en la cantidad de lixiviado a tratar. Se tienen entonces dos dinámicas distintas que afectan la cantidad de los lixiviados a tratar, una lenta que afecta el caudal medio y una rápida que afecta los caudales diarios.

Las variaciones de los caudales de lixiviados afectan de manera diferente a los sistemas de tratamiento. Algunos sistemas pueden acomodar en su proceso las variaciones en caudal, sin afectar el rendimiento, mientras que otros no. Esto puede generar la necesidad de estructuras de almacenamiento y ecualización de caudales que afectan los costos del proceso. En otros casos las economías de escala de los sistemas pueden igualmente favorecer plantas grandes; mas sin embargo, dado que los caudales medios varían lentamente, se tienen lucros cesantes del capital, al tenerse una capacidad instalada que no se utiliza durante una gran parte del tiempo. Esto hace muy onerosas las inversiones. Un sistema modular, que permita expandirse y contraerse fácilmente puede representar ventajas claras en cuanto al flujo de capital y a costos de operación y mantenimiento de los sistemas que se instalen. 


\subsection{Otras consideraciones}

En algunos casos, como en lixiviados provenientes de rellenos sanitarios en donde se depositen residuos de actividades comerciales e industriales, los lixiviados arrastran de manera disuelta cantidades considerables de compuestos orgánicos volátiles, COV. Estos compuestos, como su nombre lo indica, se disuelven en el agua pero salen fácilmente de solución por tener una alta volatilidad. Muchos de estos compuestos son de interés ambiental porque además de ser volátiles son muy tóxicos, de tal forma que el desprendimiento de éstos a la atmósfera, bien sea en las conducciones o en el proceso mismo de tratamiento puede llegar a ser una consideración ambiental de importancia. Por ejemplo, los sistemas biológicos que utilicen aire inyectado en el proceso para el suministro de oxígeno, son en sí sistemas que arrastraran junto con el aire que sale, una gran cantidad de COV que se convierten en una emisión de contaminación a la atmósfera. Lo mismo, pero en menor cuantía, puede ocurrir con los sistemas anaerobios en donde el gas que se produce dentro del reactor arrastraría igualmente los COVs fuera del sistema. Si este gas se quema, y la temperatura y el tiempo de quemado es suficiente, entonces estos COVs pueden ser destruidos en la llama. En algunos casos es necesario hacer tratamiento adicional de los gases expelidos por el sistema de tratamiento de los lixiviados. En 1997 la USA-EPA reglamentó el control de emisiones de COV a la atmósfera requiriendo que los rellenos que superaran una cantidad másica dada de emisiones de COVs deberían implementar sistemas recolección y control.

Una consideración adicional en la selección del proceso es la generación de lodos del sistema de tratamiento. La alternativa mas obvia es espesarlos, deshidratarlos y retornarlos al relleno sanitario; sin embargo, puede haber problemas con la legislación ambiental que en algunos sitios limita la disposición de lodos de plantas de tratamiento en rellenos sanitarios. En estos casos la producción de lodos por el sistema seleccionado, y su manejo posterior, se puede tornar en una variable de selección importante a la hora de escoger una tecnología. Existen grandes diferencias en la producción y manejo de los lodos que se producen entre las diferentes tecnologías.

\section{Alternativas de}

\section{TRATAMIENTO}

Una vez presentadas las principales características de los lixiviados que hay que tener en cuenta en su tratamiento, en el presente apartado se pasa a hacer una exposición de las principales tecnologías para el tratamiento de los lixiviados. Se hace primero una exposición de las principales tecnologías existentes, y luego se pasa a mirar algunos avances recientes.

Las alternativas de tratamiento de lixiviados se pueden categorizar de acuerdo a varias características como por ejemplo de acuerdo a los niveles de tratamiento que se logren con cada una de ellas, o por el tipo de contaminación que puedan remover. Vale la pena recordar que los lixiviados contienen todos los mayores grupos de contaminación conocidos como son la contaminación por patógenos, por materia orgánica, la contaminación por nutrientes, y por sustancias tóxicas. Como se mencionó en el apartado anterior, en algunos casos la remoción de uno de los grupos de contaminación se ve impedido por la presencia del otro grupo como es el caso de la remoción de la materia orgánica y los metales pesados.

No es exagerado decir, que todas las tecnologías conocidas para el tratamiento de aguas residuales se han probado para el tratamiento de los lixiviados de rellenos sanitarios. Existe una extensa literatura técnica sobre las aplicaciones de las diferentes tecnologías para el tratamiento de lixiviados. Una revisión detallada de cada tema está más allá de los alcances del presente documento. A continuación se hace un resumen de las principales tecnologías que se tienen actualmente. No se hace una discusión de la opción de tratar los lixiviados en plantas de tratamiento de aguas residuales municipales mezclando el lixiviado con el resto del agua residual de la ciudad, ya que en la mayoría de los casos estas son inexistentes en los países en desarrollo y por lo tanto no es una opción viable. Sin embargo, la experiencia muestra que cuando esta es una opción, usualmente es una opción eficiente en costos.

\subsection{Procesos anaerobios}

Las tecnologías clásicas para la remoción de materia orgánica, que como en el caso de los lixiviados es predominantemente materia orgánica disuelta, son los procesos biológicos de tratamiento. Para el caso de un lixiviado joven, en especial lixiviados de rellenos con altos contenidos de MOFBD, los consecuentemente altos contenidos de materia orgánica parecieran idealmente apropiados para la aplicación de los procesos anaerobios de tratamiento. De hecho existen numerosos reportes de trabajo de todo tipo de tecnologías anaerobias, desde las mas simples lagunas anaerobias, hasta complicados sistemas de lecho fluidizado, pasando por filtros anaerobios y reactores UASB. En términos de las reducciones de DBO se reportan muy altas eficiencias a 
cargas razonables. Usualmente se usan para llegar a niveles de tratamiento secundario, pero cuando se requieren eficiencias superiores se utilizan como pretratamiento, precediendo a sistemas aerobios como los lodos activados.

Las principales ventajas que tienen los procesos anaerobios en este contexto son la mayor simplicidad en el sistema de tratamiento y la menor producción de lodos. Esto se refleja en menores costos de inversión de capital y de operación y mantenimiento, y en menores requisitos técnicos en el personal que opera el sistema.

Sin embargo, existen varias precauciones que hay que tener en cuenta al aplicar este tipo de procesos. Los altos contenidos de amoníaco y de minerales disueltos pueden generar problemas de toxicidad para los microorganismos. Esto implicaría una remoción previa del amoníaco en caso de que este fuera el problema, o la aplicación de cargas de trabajo reducidas debido a las limitaciones en la actividad microbiana por motivo de la toxicidad.

Por otra parte los investigadores que han trabajado con los sistemas de tratamiento anaerobio para lixiviados en rellenos sanitarios coinciden en indicar una acumulación muy significativa de material inorgánico precipitado dentro del reactor y en los lodos mismos del sistema anaerobio. Como se mencionó anteriormente este es un problema práctico de gran significancia para la operatividad de los sistemas. La acumulación de material precipitado dentro del reactor termina por formar incrustaciones que limitan el volumen activo del reactor, limitan la actividad de los lodos, y taponan los sistemas de conducciones de los reactores acabando finalmente en un colapso del sistema de tratamiento, o alternativamente, en costos y complicaciones muy grandes en la operación y mantenimiento de las plantas.

Con base en estas experiencias, se concluye que es necesario bien sea hacer pre-tratamientos que minimicen los efectos de la toxicidad y/o de los materiales incrustantes, $o$, trabajar con diseños de reactores anaerobios que sean mas resistentes a éstos fenómenos.

Igualmente debe tenerse en cuenta que las variaciones de los caudales y cargas orgánicas en sistemas como los reactores UASB pueden fácilmente desestabilizar el proceso, y por lo tanto con frecuencia se puede requerir una homogenización de los caudales, si se esperan variaciones significativas en los flujos y cargas.

\subsection{Procesos Aerobios}

Los procesos aerobios al igual que los anaerobios han sido ampliamente estudiados para el tratamiento de los lixiviados de rellenos sanitarios. Existe experiencia con una gran variedad de tipos de sistemas, desde las tradicionales lagunas aireadas, hasta sofisticados sistemas que acoplan reactores biológicos con procesos de ultrafiltración con membranas. Su rango de aplicación es conocido al igual que los problemas y limitaciones que pueden surgir en su aplicación. Se utilizan cuando se requiere obtener una baja concentración de $\mathrm{DBO}$ en los efluentes. Vale la pena aclarar que como usualmente las concentraciones de DBO en los lixiviados son muy altas es relativamente fácil tener remociones porcentuales superiores al $90 \%$ en este parámetro. Sin embargo la DBO remanente puede ser todavía alta. Los costos de inversión y de operación y mantenimiento son significativamente superiores a los de los procesos anaerobios cuando los lixiviados son concentrados, como es el caso de un lixiviado joven, por lo que se logran mejores relaciones beneficio / costo cuando se utilizan para tratar lixiviados con concentraciones medias o bajas de DBO. Por esta razón, y dependiendo de las exigencias del vertimiento, se usan preferencialmente como posttratamiento a los sistemas anaerobios, o para lixiviados viejos con bajos niveles de DBO.

En los reportes operativos se mencionan problemas con la generación de espumas, con la precipitación de hierro, y en el caso de los lodos activados, problemas para aceptar altas variaciones en las cargas hidráulicas y orgánicas que caracterizan a los lixiviados, como ya se ha mencionado anteriormente. Esto último puede implicar que los sistemas requieran tanques de ecualización de caudales como parte del tratamiento. Igualmente, y dependiendo de la forma de operación del proceso, se tiene una alta generación de lodos residuales, en mayor cantidad que los procesos anaerobios, que es necesario procesar aumentando los costos de inversión y de operación y mantenimiento. Por otra parte, en casos en donde los lixiviados traigan cantidades importantes de COVs, el aire que se usa en el proceso de la aireación del tanque biológico debe ser tratado a su vez para remover los COV que se arrastran. Esto igualmente hace más compleja la operación de los sistemas de tratamiento y aumenta los costos.

Por la naturaleza misma del proceso que se tiene, la operación de un proceso aerobio requiere mayor capacidad técnica por parte del operador, al igual que mayor necesidad de mantenimiento de equipos.

\subsection{Sistemas Naturales}

Los sistemas naturales, lagunas y humedales artificiales, también se han propuesto como alternativas para el tratamiento de lixiviados. Tienen la ventaja de la simpli- 
cidad en su operación, y la posibilidad de lograr diferentes niveles de tratamiento, desde un pretratamiento, hasta un tratamiento terciario en caso de necesitarse. La combinación de las lagunas y los humedales puede manejar adecuadamente muchos de los problemas que en otras tecnologías aparecen como son la acumulación de precipitados, la formación de espumas, la toxicidad a los microorganismos, y las variaciones en cargas hidráulicas y orgánicas. Esto se logra al tener tiempos de retención hidráulica muy altos y volúmenes de procesos igualmente grandes, que permiten acomodar variaciones en caudal, acumulaciones de precipitados, junto con una baja producción de gases y por lo tanto de espumas. Desde el punto de vista de costos en valor presente, la tecnología a probado ser muy competitiva al compararse con otras alternativas. Es importante mencionar que los análisis financieros deben tener en cuenta el valor presente de los costos de capital y de operación y mantenimiento de los sistemas. De otra forma se puede llegar a conclusiones erradas con respecto al costo real por volumen de lixiviado tratado en un relleno sanitario. Si bien es cierto que los costos de capital pueden llegar a ser similares en sitios donde el costo del terreno es alto, los bajos costos de operación y mantenimiento son una de las principales razones de los bajos costos en valor presente. En aplicaciones en donde el costo de la tierra no es muy alto, o donde las zonas de amortiguamiento del relleno sanitario se pueden usar en el proceso, la tecnología presenta costos de inversión inicial substancialmente menores que otras alternativas.

La principal desventaja que se tiene con estos sistemas es la cantidad de terreno que requiere para localizar los procesos. Sin embargo, por la naturaleza misma de los diseños de los rellenos sanitarios, en donde hay necesidad de tener áreas de amortiguamiento visual, de ruido, y de olores, estas áreas que usualmente están localizadas en los alrededores del relleno, podrían utilizarse como parte de los sistemas naturales de tratamiento; en especial en el caso de los humedales.

En el caso de los humedales artificiales, su aplicación al tratamiento de los lixiviados es nueva, con experiencias en Estados Unidos y en Europa principalmente.

\subsection{Evaporación}

La utilización de la evaporación como sistema de tratamiento de lixiviados es una aplicación nueva, al igual que los humedales. En ella se utiliza la energía que se tiene en el biogás del relleno sanitario en evaporar el lixiviado por calentamiento. Existen varios tipos de tecnologías ya desarrolladas para lograr el objetivo. Las tecnologías existentes permiten lograr el control del total de emisiones de lixiviados del relleno sanitario, quedando un lodo que se dispone nuevamente en el relleno. La experiencia y los cálculos de producciones de gas y lixiviados en los rellenos sanitarios indican que se tiene gas en exceso para suplir las necesidades energéticas de evaporación del lixiviado. Dependiendo del tipo de lixiviado en algunos casos existe la necesidad de hacer un post-quemado de la mezcla gas-vapor de agua que sale del evaporador para lograr la destrucción de emisiones de COVs que se arrastran durante el proceso de evaporación, de tal manera que la cantidad requerida de biogás se aumenta con respecto a los cálculos termodinámicos normales. Sin embargo, una vez quemados los COVs las emisiones del proceso se limitan a vapor de agua y a un lodo espesado.

Algunas de las tecnologías utilizan de manera directa la energía que se genera al quemar el gas con el objetivo central de evaporar el lixiviado, lo que se denomina vaporización del gas, mientras que otras tecnologías pueden utilizar el calor residual que generan motores de combustión o turbinas, que utilizan el biogás para generar potencia mecánica, que a su vez se puede usar para la generación eléctrica. De esta manera, se están logrando llevar a cabo no solamente el aprovechamiento del gas para la conversión a energía eléctrica, sino el tratamiento de los lixiviados, solucionando los dos principales problemas que tienen los rellenos sanitarios: emisiones de gases y de lixiviados. Esta es talvez la principal ventaja que se tiene con la tecnología de la evaporación y que no se logra con ninguno de las otras alternativas de una manera simultánea como en este caso.

Con la importancia mundial que están tomando el fenómeno de los gases invernadero y el cambio climático, la destrucción térmica del metano de los rellenos sanitarios se ha identificado como una de las maneras mas efectivas en costos para obtener reducciones en las emisiones globales de metano. Vale la pena mencionar que el metano es aproximadamente 15 veces mas activo que el dióxido de carbono en la retención de las radiaciones infrarrojas causantes del fenómeno del calentamiento global. Por esta razón y por la necesidad de controlar las emisiones de COVs en los rellenos sanitarios el quemar el metano es cada vez más una práctica común en los países desarrollados. Una vez se ha quemado el metano, la energía para la evaporación se encuentra entonces disponible.

Otras de las ventajas que con frecuencia se mencionan en favor de la tecnología de la evaporación son la simplicidad tecnológica de los equipos, y los bajos costos comparativos con otras tecnologías similares. 
La tecnología de la evaporación también ha reportado problemas operativos, que en algunos casos ya se sabe como solucionarlos, pero que sin embargo vale la pena mencionar. En general son problemas similares a los reportados para otras de las tecnologías citadas anteriormente, como es el caso de la formación de espumas por la turbulencia generada en el proceso de evaporación, el incrustamiento de precipitados en el sistema, y el arrastre de COVs. Igualmente cuando los lixiviados son jóvenes y existen altas concentraciones de ácidos grasos volátiles y amoníaco, y dependiendo del $\mathrm{pH}$ al cual se realice la evaporación, estos compuestos se pueden arrastrar junto con el vapor de agua. En algunos casos se ha propuesto realizar ajustes de $\mathrm{pH}$ para minimizar el arrastre de los ácidos y/o del amoníaco. En otros casos se han propuestos sistemas de evaporación múltiple que en una etapa se controlan las emisiones del amoníaco, mientras que en la otra las emisiones de ácidos grasos. De hecho varias de las tecnologías existentes permiten hacer ajustes de $\mathrm{pH}$ durante el proceso de evaporación.

\subsection{Recirculación de los Lixiviados}

La recirculación de los lixiviados se ha propuesto desde hace varios años como una alternativa para su tratamiento. Más recientemente se conoce su uso como la tecnología del relleno biorreactor, que en la actualidad se está estudiando en detalle en los Estados Unidos con apoyo de la EPA para elucidar varias preguntas que aun persisten sobre el proceso. Se pretende utilizar el relleno sanitario como un gran reactor anaerobio de tal manera que dentro del mismo relleno se logre la conversión a metano de los ácidos grasos que están presentes en el lixiviado. Al recircular los lixiviados se logra un aumento en la humedad de los residuos dispuestos, que a su vez genera un aumento de la tasa de producción de gas metano en el relleno. Una vez los ácidos grasos han sido metanizados, el pH del lixiviado aumenta, y al aumentar el $\mathrm{pH}$ la solubilidad de los metales disminuye de tal forma que se logra una disminución de los metales en solución que son transportados por el lixiviado. De esta manera se logra una reducción significativa tanto de la DBO como de los metales que finalmente arrastra el lixiviado. Usualmente se considera que el nivel de tratamiento alcanzado es el de pretratamiento, siendo necesario algún tipo de tratamiento posterior que dependerá de los requisitos de los permisos de vertimiento en cada caso. Las experiencias indican que entre 3 y 10 años se debe recircular un lixiviado para generar los efectos de tratamiento requeridos.

A la recirculación del lixiviados con frecuencia se le imputan otros beneficios adicionales a su efecto en el pretratamiento de los lixiviados como son: aumento en las tasas de producción de biogás en el relleno sanitario, maximización de la producción de gas por tonelada de residuo dispuesta, aumento de las tasas de estabilización y asentamientos en el relleno. Esta última a su vez genera ventajas como son el aumento de la capacidad del relleno por la ganancia asociada de volumen, y la disminución en las actividades de post-clausura del relleno. En el caso de los residuos sólidos municipales de los países en desarrollo, en donde la humedad intrínseca de los residuos es superior a la de los países desarrollados, usualmente las tasas de producción de gas son superiores a las que se reportan en los rellenos sanitarios de países desarrollados. Dentro de esta lógica, es de esperarse que los beneficios adicionales de la recirculación en los rellenos sanitarios no sean tan notorios en países en vías de desarrollo como si lo son en los países desarrollados en donde las tasas de producción de gas se ven severamente limitadas por la humedad.

Adicionalmente, por el aumento de la humedad y la tasa de generación de gas, la recirculación de los lixiviados en el relleno sanitario puede generar aumentos significativos de las presiones de internas de los fluidos, gases y líquidos, que comprometan la estabilidad estructural de los taludes. Este efecto puede ser más notorio en los residuos húmedos de países en desarrollo que en los secos de los países desarrollados. Los efectos de la humedad en las conductividades hidráulicas de los gases y los líquidos en un medio no saturado como un relleno sanitario, obedecen a relaciones altamente no lineales, en donde pequeñas variaciones de la humedad pueden generar grandes variaciones en la conductividad. Estas variaciones en la conductividad de los gases y líquidos dentro de la matriz del relleno, se reflejan en aumentos de las presiones internas en el relleno.

Es necesario mirar con cuidado los aspectos de seguridad geotécnica en los rellenos sanitarios cuando se considere el uso de la recirculación de los lixiviados como un método de pretratamiento. Esto implica cuidados especiales en términos de la instrumentación geotécnica del relleno, y en los sistemas de drenaje y evacuación de líquidos y gases. Con frecuencia esta instrumentación adicional, al igual que los requisitos adicionales de drenaje tanto de lixiviados como de gases aumentan significativamente el costo de los sistemas.

\subsection{Sistemas de Membranas}

La tecnología del tratamiento de aguas utilizando membranas es una tecnología de rápido desarrollo en la última década. Con mayor frecuencia se observan más aplicaciones de las membranas en el tratamiento de todo 
tipo de efluentes, incluyendo obviamente los lixiviados de rellenos sanitarios. Se encuentra en la literatura aplicaciones de la microfiltración, la ultrafiltración, la nanofiltración, la ósmosis inversa, la ósmosis directa e inclusive la pervaporación al tratamiento de los lixiviados, bien sea de manera directa, o acoplada a otro tipo de proceso de tratamiento. Por ejemplo, se observa que tanto la microfiltración como la ultrafiltración se han acoplado a procesos biológicos de tratamiento aerobio, en reemplazo de los sedimentadores, tanto para la remoción de DBO, como para la nitrificación del amoníaco. Igualmente se encuentran reportes de la aplicación en serie de procesos de ósmosis inversa con procesos de precipitación-cristalización y nanofiltración para la remoción de sustancias precipitables de lixiviados con alto contenido de sólidos disueltos inorgánicos. De la misma manera se tienen reportes de la aplicación directa de la ósmosis inversa, y la ósmosis directa en el tratamiento de lixiviados. A continuación se hará una breve reseña de estos procesos.

\section{Biorreactores con Membrana, MBR:}

Los biorreactores con membrana se utilizan de la misma manera como se utilizan los sistemas biológicos de tratamiento, siendo la principal diferencia la sustitución del sedimentador como sistema de separación sólidolíquido por un sistema de micro o ultrafiltración. Esto puede tener ventajas en términos de la disminución del volumen de tanque del reactor biológico, mas sin embargo, introduce complicaciones adicionales en la operación de los sistemas ya que los módulos de membranas son más complicados de operar y mantener que un sedimentador. Igualmente se logran aumentos significativos en la cantidad de biomasa que se tiene dentro de los reactores, pero al mismo tiempo se puede perder eficiencia en la transferencia de masa en la aireación, de tal manera que se aumentan los costos de energía por este sentido. Análisis recientes indican que, de hecho, lo que se gana en costos por la reducción del tamaño de los tanques de aireación, se pierde por el aumento en costos asociados a los equipos de aireación, al igual que el aumento de costos de operación.

Igualmente se reportan disminuciones en el flux a través de la membrana por procesos de taponamiento. Es necesario considerar dentro de la selección de la tecnología la garantía de un suministro adecuado de reemplazo de membranas, al igual que incluir estos costos dentro de los cálculos financieros de operación y mantenimiento de este tipo de sistemas.

Las eficiencias que se han reportado tanto para las aplicaciones en las cuales se utiliza el proceso biológico para la oxidación del amoníaco, como en aquellas en las cuales se busca remover la DBO, son excelentes siendo ésta su principal ventaja.

\section{Osmosis INVERSA}

En general se reportan unos excelentes rendimientos de la tecnología para la remoción de la mayoría de los contaminantes. Igualmente se observa que las aplicaciones han sido para lixiviados con concentraciones de DBO relativamente bajas, menores a $1000 \mathrm{mg} / \mathrm{l}$, es decir, lixiviados viejos, o lixiviados a los cuales se les ha realizado un pretratamiento previo. Se habla de concentraciones relativamente bajas, porque dentro de los rangos de DBO de los lixiviados de rellenos sanitarios de paises en desarrollo se tienen concentraciones en los lixiviados jóvenes del orden de las decenas de miles de miligramos por litro, es decir, entre 10 y 40 veces mas concentrados que los que se reportan en la literatura técnica. Se debe entonces tener cautela en la aplicación de la tecnología de manera directa a lixiviados jóvenes, especialmente de aquellos que se encuentran en los países en desarrollo. Otra ventaja que se reporta con frecuencia en el caso de la ósmosis inversa son los bajos consumos energéticos que requiere la tecnología cuando se compara con otras tecnologías como la oxidación biológica o la evaporación.

Se han reportado problemas de colmatación asociados a la precipitación del calcio y el hierro en las membranas, obligando a la incorporación de sistemas de pretratamiento que minimicen estos efectos. Esto se ha logrado de diversas maneras, desde la aplicación de sustancias químicas que modifiquen las condiciones bajo las cuales ocurre la precipitación, hasta la incorporación de nuevos procesos tales como la osmosis directa o la precipitación-cristalización. Igualmente se ha reportado una disminución significativa del flux a través de la membrana con el aumento de la concentración de DBO en el concentrado, lo que conlleva la necesidad de tener varios sistemas trabajando en serie para optimizar el proceso. En la terminología de las membranas se denomina el permeado lo que pasa la membrana y el concentrado lo que queda retenido.

La tecnología es intensiva en cuanto a la operación y mantenimiento necesario, por la necesidad de hacer lavados, limpiezas y reemplazos de las membranas, consideración que debe tenerse en cuenta en el momento de seleccionar la tecnología.

\section{Discusión}

Como se puede observar, el tratamiento de los lixiviados de los rellenos sanitarios es un problema difícil de 
atacar, tal vez, sin exageración, uno de los problemas más desafiantes en la ingeniería del tratamiento de las aguas residuales. Esta apreciación quizás ayuda a explicar la gran cantidad de tecnologías y de investigación que se ha realizado, y aún se realiza, alrededor del tema. En general puede decirse que todavía hay mucho campo para la innovación. Tal vez la solución final y racional consista en no producir los lixiviados, o al menos en producir lixiviados de características mucho menos contaminantes. Sin embargo esta solución se podrá dar cuando se mire de una manera global el flujo de materiales en la sociedad y se internalizen los costos ambientales en todo el ciclo de los materiales, desde su producción, transformación, distribución, uso y descarte. Mientras tanto es muy probable que los lixiviados continúen siendo un problema apremiante de la sociedad. En la Tabla 2 se observa un resumen de las principales características y consideraciones que se deben tener en cuenta en la comparación de las tecnologías que se presentaron anteriormente.

En general se puede observar que los problemas de acumulación de precipitados, emisiones de COV's, toxicidad a los microorganismos, formación de espumas afectan de manera similar a los procesos biológicos in- tensivos. Esto ha hecho que las aplicaciones sean usualmente sistemas híbridos en los cuales se logre unos pretratamientos iniciales en donde se acondicione el lixiviado para minimizar los efectos mencionados anteriormente. De hecho, se puede hablar de un tren de tratamiento "clásico" como se observa en la Figura 1. Allí se tiene ecualización de caudales y calidades en un tanque inicial para minimizar los efectos de variaciones de cargas hidráulicas y orgánicas en los procesos posteriores. Luego un sistema físico-químico de remoción de constituyentes precipitables, que incluye parte de los metales pesados, utilizando una elevación del $\mathrm{pH}$ para lograr su precipitación y su remoción por sedimentación. Posteriormente se baja nuevamente el pH para ajustarlo al tratamiento biológico utilizando ácido fosfórico- que a su vez suministra el fósforo que le hace falta al sistema biológico-. El sistema biológico incluye una primera etapa anaerobia que tiene altas eficiencias a bajos costos, relativos, y un pulimento posterior en una segunda etapa aerobia que permite bajas concentraciones en el efluente en términos de DBO, e inclusive la remoción de amoníaco a través de la nitrificacióndesnitrificación biológica. En algunos casos la remoción del amoníaco se realiza en las etapas del pretrata-

TABLA 2

Comparación entre Tecnologías para el Tratamiento de Lixiviados (MANEJo de CARACterísticas PRoblemáticas de LOS LIXIVIADOS)

\begin{tabular}{|c|c|c|c|c|c|c|c|c|}
\hline \multirow[t]{2}{*}{ ITEM } & \multirow[t]{2}{*}{ Problemas CON } & \multicolumn{7}{|c|}{ Tecnología } \\
\hline & & AERoBio & ANAEROBio & EVAPORACIÓN & RECIRCULACIÓN & Membranas & $\begin{array}{l}\text { Sistemas } \\
\text { Naturales }\end{array}$ & $\begin{array}{l}\text { TRATAMIENTO } \\
\text { EN PTAR }\end{array}$ \\
\hline 1 & $\begin{array}{l}\text { Formaciónde } \\
\text { Precipitados }\end{array}$ & ++ & +++ & + & ++ & +++ & + & No \\
\hline 2 & $\begin{array}{l}\text { Toxicidad a los } \\
\text { microorganismos }\end{array}$ & ++ & ++ & No & No & No (1) & + & Potencial \\
\hline 3 & $\begin{array}{l}\text { Formación de } \\
\text { espumas }\end{array}$ & +++ & + & ++ & $+(1)$ & Variable (2) & No & Baja \\
\hline 4 & Emisión de $\mathrm{COV}$ & +++ & + & ++ & + & Variable (2) & + & Baja \\
\hline 5 & $\begin{array}{l}\text { Sensibilidad a } \\
\text { variaciones de caudal }\end{array}$ & ++ & ++ & + & + & + & No & No \\
\hline 6 & $\begin{array}{l}\text { Producción y manejo } \\
\text { de lodos }\end{array}$ & +++ & + & + & No & + & & No \\
\hline 7 & $\begin{array}{l}\text { Requerimientos } \\
\text { de área }\end{array}$ & Baja & Baja & Muy baja & Baja & Baja & Alta & No \\
\hline
\end{tabular}

(1) Pueden formarse en los tanques de almacenamiento

(2) Si los sistemas son aerobios, la problemática puede ser alta.

${ }^{(+)}$Una cruz significa como afecta negativamente la característica al proceso en cuestión. Entre más cruces más negativamente lo afecta. 
miento aprovechando el hecho de que al subir el $\mathrm{pH}$ para la precipitación igualmente se puede volatilizar el amoníaco. Esta decisión depende de la acidez del sistema y por lo tanto de los costos relativos de las sustancias químicas para subir y bajar el $\mathrm{pH}$ nuevamente. Finalmente para la remoción final de patógenos se tiene un sistema de desinfección para los cuales existen numerosas tecnologías alternativas.

Se puede observar igualmente en las Tabla 2 y 3 que tecnologías como la evaporación pueden lograr reducciones similares y/o superiores a los sistemas combinados como los presentados en la Figura 1 teniendo me-

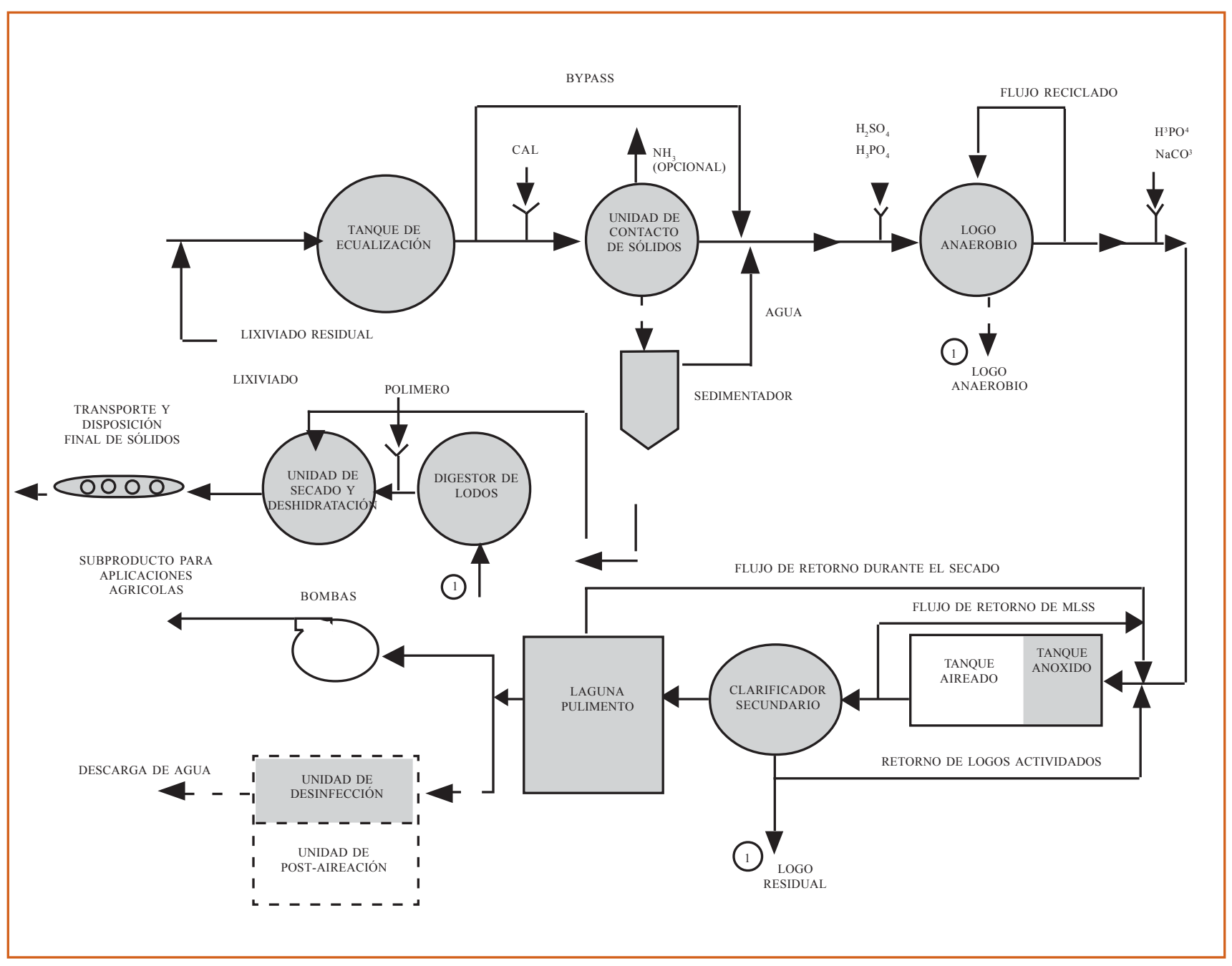

Figura 1. Esquema de Tren Tradicional de Tratamiento de Lixiviados

nos problemas operacionales, menores emisiones de lodos, y de acuerdo a comparaciones realizadas en países desarrollados, aparentemente mucho menores costos de tratamiento.

La recirculación de los lixiviados, o como lo denominan más recientemente: los rellenos-biorreactores- igualmente comparten muchas de las ventajas de las otras tecnologías más sin embargo, como ya se mencionó, existen serios cuestionamientos sobre sus efectos sobre la estabilidad geotécnica del relleno, lo que limita su aplicación a situaciones donde se pueda comprobar la estabilidad, y adicionalmente sobre la necesidad posterior de pulir finalmente el lixiviado recirculado y pretratado.

Los sistemas naturales por su parte, pueden tener igualmente excelentes remociones y un buen manejo de los principales problemas asociados al tratamiento de los lixiviados. Esto se logra básicamente usando altos tiempos de retención hidráulica en los sistemas, suficientes 
TABLA 3

Comparación entre TeCnologías para el Tratamiento de Lixiviados

(COMPLEJIDAD TECNOLÓGICA)

\begin{tabular}{|c|c|c|c|c|c|c|c|c|}
\hline \multirow[t]{2}{*}{ Iтем } & \multirow[t]{2}{*}{ Problemas con } & \multicolumn{7}{|c|}{ COMPLEJIDAD TECNOLÓGICA } \\
\hline & & AERoBio & ANAERoBio & EVAPORACIÓN & RECIRCULACIÓN & Membranas & $\begin{array}{l}\text { SisTEMAS } \\
\text { NATURALES }\end{array}$ & $\begin{array}{l}\text { TRATAMIENTO } \\
\text { EN PTAR }\end{array}$ \\
\hline 1 & $\begin{array}{l}\text { Necesidadde } \\
\text { insumos químicos }\end{array}$ & $+++(1)$ & $+++\quad(1)$ & $++(2)$ & - & $+++\quad(1)$ & - & - \\
\hline 2 & $\begin{array}{l}\text { Necesidad de in- } \\
\text { sumos operacio- } \\
\text { nales (v. gr. mem- } \\
\text { branas) }\end{array}$ & + & + & + & + & $+1+$ & - & - \\
\hline 3 & $\begin{array}{l}\text { Necesidad de su- } \\
\text { ministro de partes }\end{array}$ & ++ & - & + & + & $+1+$ & - & + \\
\hline 4 & $\begin{array}{l}\text { Suministro de } \\
\text { Energía Eléctrica }\end{array}$ & $+1+$ & $+(4)$ & $-(5)$ & + & $+1+$ & $+(3)$ & + \\
\hline 5 & $\begin{array}{l}\text { Complejidad } \\
\text { Operacional }\end{array}$ & $+1+$ & + & + & + & $+1+$ & - & - \\
\hline
\end{tabular}

(1) Requiere un extenso pretratamiento

$\left({ }^{2}\right)$ Puede requerir pretratamiento, algunos sistemas usan sustancias para control de $\mathrm{pH}$, espumas.

(3) Puede requerir si hay necesidad de bombear el lixiviado. Usualmente no requiere.

${ }^{(4)}$ Puede requerir en el sistema de pretratamiento

$\left({ }^{5}\right)$ Puede llegar a ser autosuficiente. Algunas tecnologías así están diseñadas.

volúmenes de almacenamiento de precipitados y lodos, bajas tasas de reacción, y mayores áreas que las tecnologías anteriores. Poseen igualmente el gran atractivo de la sencillez y simplicidad tecnológica que los puede hacer apropiados en situaciones donde la complejidad afecte la sostenibilidad operativa.

Finalmente los sistemas de membranas aparecen como una alternativa viable, con altos rendimientos operacionales en la limpieza del lixiviado, pero comparten en gran medida la complejidad tecnológica que se le atribuye a los sistemas clásicos. Con frecuencia requieren de pretratamientos similares a los presentados en la Figura 1.

No se puede llegar a generalizaciones sobre la existencia de una tecnología óptima en todos los casos pues las condiciones de cada sitio pueden variar e influenciar significativamente la selección. Se puede citar por ejemplo, la disponibilidad de terrenos, de energía eléctrica, de suministros de químicos, de partes de repuesto, o de personal calificado para la operación. Si los terrenos son costosos, o no se encuentran disponibles en los alrededores del relleno sanitario, muy probablemente la tecnología de los humedales artificiales no es la más conveniente. Si no existe suministro de energía eléctrica, sistemas que requieran de equipos mecánicos como aireadores, bombas, mezcladores, compresores, prensas, centrífugas etc. seguramente entraran en desventaja con tecnologías mas sencillas o autosuficientes como la evaporación o los sistemas naturales. La complejidad tecnológica de la operación debe igualmente considerarse. Una gran ciudad probablemente pueda contar con el personal capacitado para la operación de sistemas que requieran dosificaciones precisas de químicos, mantenimiento de partes móviles, reemplazo de partes claves, limpieza de equipos, etc. más este no puede ser el caso de una población intermedia; lo que afectaría necesariamente la sostenibilidad tecnológica del sistema de tratamiento en el mediano y largo plazo.

Finalmente una variable decisiva son los costos. La información disponible indica que en general las tecnologías alternativas como la evaporación y los sistemas de humedales son significativamente más económicos que los sistemas como los presentados en la Figura 1 cuando se consideran los costos totales en valor presente neto de todos los procesos, y se expresan en costos por unidad de lixiviado tratado. Algunos reportes de USA inclusive indican que los costos de los humedales pueden llegar a ser comparables con los costos del tratamiento en PTAR municipal, que usualmente es de lejos, la opción más eficiente en costos. Debe tenerse 
sinembargo mucho cuidado con esta aseveración pues los datos disponibles son en condiciones operacionales y con lixiviados usualmente de países desarrollados que como ya se mencionó son significativamente diferentes a los de países en vías de desarrollo.

TABLA 4

Comparación entre TeCnOlogías para el Tratamiento de Lixiviados

(RENDIMIENTO de REMOLICIÓN)

\begin{tabular}{|c|c|c|c|c|c|c|c|c|}
\hline \multirow[t]{2}{*}{ ITEM } & \multirow[t]{2}{*}{ Problemas con } & \multicolumn{7}{|c|}{ ReNDIMIENTOS } \\
\hline & & AERoBio & ANAEROBIO & EVAPORACIÓN & RECIRCULACIÓN & MEMBRANAS & $\begin{array}{l}\text { Sistemas } \\
\text { NATURALES }\end{array}$ & $\begin{array}{l}\text { TRATAMIENTO } \\
\text { EN PTAR }\end{array}$ \\
\hline 1 & $\begin{array}{l}\text { Demanda Bioquí- } \\
\text { mica de Oxígeno }\end{array}$ & Muy altos & Altos & Muy altos & Intermedios & Muy altos & Muy altos & Muy altos \\
\hline 2 & Nutrientes & Altos ${ }^{(1)}$ & Muy bajos & Muy altos & Bajos & No ${ }^{(1)}$ & No & Variables ${ }^{(4)}$ \\
\hline 3 & Metales & Intermedios $^{(2)}$ & Altos & Muy altos & Intermedios & Altos & Altos & Altos \\
\hline 4 & $\begin{array}{l}\text { Compuestos Orgá- } \\
\text { nicos Volátiles }(\mathrm{COV})\end{array}$ & Altos ${ }^{(3)}$ & + & Muy altos & + & No ${ }^{(1)}$ & + & Variables ${ }^{(5)}$ \\
\hline 5 & Patógenos & Bajos & Bajos & Muy altos & Bajos & Muy altos & Variables $^{(4)}$ & Variables $^{(4)}$ \\
\hline
\end{tabular}

(1) Pueden ser altos o bajos dependiendo del diseño

${ }^{2}$ ) Cuando hay pretratamiento pueden tener remociones muy altas

$\left({ }^{3}\right)$ La remoción se hace por arrastre en el tanque de aireción. Este genera problemas d impacto ambiental

$\left({ }^{4}\right)$ Puede ser muy altos si asi se requiere

$\left(^{5}\right)$ Puede generar problemas en las conducciones

TABLA 5

CompaRACIÓN DE Costos

\begin{tabular}{|l|c|}
\hline \multicolumn{1}{|c|}{ TRATAMIENTO } & COSTO US $\$ \mathrm{~m}^{3}$ \\
Proceso aeróbico con remolición de nitrógeno & 20 \\
Osmosis Inversa en dos etapas & $7-10$ \\
Proceso Biológico + Carbón Activado + Precipitación & $25-35$ \\
Proceso biológico + Osmosis Inversa + Evaporación del Concentrado & $35-40$ \\
Evaporación & 5 \\
Humedales & 1 \\
\multicolumn{1}{|c|}{ ConSUMO ENERGÉTICO } & CANTIDAD \\
Osmosis Inversa - Nanotración & $8,5 \mathrm{Kwh} / \mathrm{m}^{3}$ \\
Evaporación al vacío & $12 \mathrm{Kwh} / \mathrm{m}^{3}$ \\
\hline
\end{tabular}

Nota: Estos son costos reportados en la literatura internacional para países desarrollados.Debe tenerse cuidado al usarlos. Se presentan como punto de referencia.

\section{REFERENCIAS}

1. Giraldo E., "Manejo Integrado de Residuos Sólidos

Urbanos", 1997. 